\title{
p-Anisaldehyde Exerts Its Antifungal Activity Against Penicillium digitatum and Penicillium italicum by Disrupting the Cell Wall Integrity and Membrane Permeability
}

\author{
Jinxin Che ${ }^{1,2}$, Xiumei Chen ${ }^{1}$, Qiuli Ouyang ${ }^{1}$, and Nengguo Tao ${ }^{1 *}$ \\ 'School of Chemical Engineering, Xiangtan University, Xiangtan 411105, Hunan, P.R. China \\ ${ }^{2}$ Postdoctoral Station of Chemical Engineering and Technology, Xiangtan University, Xiangtan 411105, Hunan, P.R. \\ China
}

\begin{abstract}
Penicillium digitatum and $P$. italicum are the two important postharvest pathogens in citrus, causing about $90 \%$ of the total loss of citrus fruit during storage and transportation. Natural fungicides such as essential oils have been widely used instead of chemical fungicides for preventing and controlling postharvest diseases. In this research, $p$-anisaldehyde exhibited a strong inhibitory effect on $P$. digitatum and $P$. italicum, with the minimum inhibitory concentration and minimum fungicidal concentration values of both being $2.00 \mu \mathrm{l} / \mathrm{ml}$. Additionally, $p$-anisaldehyde visibly inhibited both the green mold and blue mold development of citrus fruits inoculated with $P$. digitatum and $P$. italicum. The mycelia morphologies of these pathogens were greatly altered, and the membrane permeability and cell wall integrity of mycelia were severely disrupted under $p$-anisaldehyde treatment. These results suggest that the antifungal activity of $p$-anisaldehyde against $P$. digitatum and $P$. italicum can be attributed to the disruption of the cell wall integrity.
\end{abstract}

Keywords: p-Anisaldehyde, antifungal activity, Penicillium digitatum, Penicillium italicum, inhibitory mechanism

Received: November 15, 2019 Accepted: March 6, 2020

First published online: March 09, 2020

* Corresponding author Phone: +86-731-58298173 Fax: +86-731-58293549 E-mail: nengguotao@126.com

pISSN 1017-7825 eISSN 1738-8872

Copyright(C) 2020 by The Korean Society for Microbiology and Biotechnology

\section{Introduction}

Citrus fruit is one of the most commercially important agricultural products in the world [1], with global production exceeding 140 million tons in 2016 (FAO, 2016). The susceptibility of citrus to postharvest diseases results in wastage and deterioration and causes serious economic losses, and most of these diseases are caused by fungal pathogens $[2,3]$. Penicillium digitatum and P. italicum are the main pathogens of two important postharvest diseases known as green mold and blue mold, which cause about $90 \%$ of the total economic loss of citrus fruit during storage and transportation $[4,5]$. Nowadays, many chemical fungicides are being widely used to control these postharvest diseases and these include fludioxonil, imazalil, and pyrimethanil [6]. Nevertheless, with the long-term and repeated use of such fungicides, their effectiveness has been gradually weakened by the proliferation of resistant fungus strains. Moreover, consumers are becoming increasingly concerned over food safety and residual contamination by fungicides $[7,8]$. In this regard, plant essential oils as natural fungicides are generally recognized as safe. Over the past decade, many such essential oils could be considered as biodegradable and safe, natural alternatives to chemical fungicides due to their broad spectrum of fungicidal activities against postharvest pathogens [9-11].

Among the essential oils, $p$-anisaldehyde is a naturally occurring, fragrant phenolic compound that is primarily isolated from anise, cumin, fennel, and garlic $[12,13]$, which has been widely used for the production of antimicrobial drugs in the pharmaceutical industry [14]. In recent studies, $p$-anisaldehyde showed strong antimicrobial activity against many microorganisms including Staphylococcus aureus [15], Candida [12], and Saccharomyces cerevisiae [16]. However, knowledge about the antifungal activity of $p$-anisaldehyde against $P$. digitatum and $P$. italicum in citrus fruits was rather limited.

The purpose of this study was to investigate the antifungal efficiency of $p$-anisaldehyde on the mycelial growth of $P$. digitatum and $P$. italicum and to reveal the possible mechanism involved.

\section{Materials and Methods}

Microorganisms, Fruits and Chemicals

P. digitatum (Pds-01) and P. italicum (Pis-01) strains were isolated from infected Satsuma mandarin fruit (Citrus unshiu Marc) and preserved in the Department of Biotechnology and Food Engineering, Xiangtan University, 
Hunan, China.

Fruits of Satsuma mandarin (Citrus unshiu Marc. cv. Miyagawa Wase) used for in vivo assay were picked in a local orchard near Xiangtan University, China, in October 2018. The citrus fruits for experiment were defect-free and with similar size and the same maturity level.

$p$-Anisaldehyde ( $>98 \%$ ) was purchased from Aladdin (China). Propidium iodide and an alkaline phosphatase (AKP) kit were purchased from Solarbio Science and Technology Co., Ltd., Beijing, China. Commercial wax (SP1) was obtained from Bo Cheng Chemical Engineering Co., Ltd (China). Other chemicals were of analytical grade and purchased from Xilong Chemical Co., Ltd. (China).

\section{Effects of $p$-Anisaldehyde on Mycelial Growth}

The effects of $p$-anisaldehyde on the mycelial growth of $P$. digitatum and $P$. italicum were evaluated by the agar dilution culture method [4]. The culture medium contained $20 \mathrm{ml}$ potato dextrose agar (PDA) supplied with final concentrations of $p$-anisaldehyde $(0.00,0.12,0.25,0.50,1.00,2.00$ and $4.00 \mu \mathrm{l} / \mathrm{ml})$. A mycelial agar plug (diameter $=6 \mathrm{~mm}$ ) from a 7-day-old culture of $P$. digitatum or P. italicum was inoculated into the PDA culture above. The plates were incubated for $5 \mathrm{~d}$ at ambient temperature $\left(24 \pm 1^{\circ} \mathrm{C}\right)$. The colony zone diameters were measured per $24 \mathrm{~h}$. All tests were performed in triplicate.

The inhibitory rate of $p$-anisaldehyde was calculated as follows:

$$
\text { Inhibitory rate }(\%)=\frac{\text { Diameter of CK }- \text { Diameter of treatment }}{\text { Diameter of CK }} \times 100 \%
$$

The lowest concentration that completely prevented the growth of P. digitatum or P. italicum after $2 \mathrm{~d}$ of cultivation was considered as the minimum inhibitory concentration (MIC). The minimum fungicidal concentration (MFC) was defined as the concentration that completely inhibited pathogen growth after $4 \mathrm{~d}$ [17].

\section{Effects of $p$-Anisaldehyde on Disease Incidence}

Citrus fruits were treated according to the previously described method [18]. In detail, citrus fruits were divided into 3 groups. Each group was composed of three repeats and each repeat included ten citrus fruits. For each surface-sterilized citrus fruit, two wounds (length of $5 \mathrm{~mm}$ and depth of $5 \mathrm{~mm}$ ) were made around the fruit equator evenly using a scalpel, with each wound subsequently inoculated with $20 \mu \mathrm{l}$ spore suspension, and left to air-dry. The $P$. digitatum and $P$. italicum spore suspensions were adjusted to $1 \times 10^{8} \mathrm{CFU} / \mathrm{ml}$. The inoculated fruits were sprayed with $p$-anisaldehyde wax at MFC or $10 \times$ MFC, then left to air-dry. The CK was sprayed with wax only. The treated fruits were stored in plastic boxes at $25 \pm 2^{\circ} \mathrm{C}$ with $85 \sim 90 \%$ relative humidity, and the disease incidence was measured each day. The inoculated fruits with wax were used as a control.

The disease incidence rate was measured by counting the number of rotting wounds using the following formula:

$$
\text { Decay incidence rate }(\%)=\frac{\text { Number of rotten wounds }}{\text { Number of total wounds }} \times 100 \%
$$

\section{Scanning Electron Microscopy (SEM)}

The SEM analysis was performed according to our previous method [17]. The mycelia of $P$. digitatum and P. italicum were obtained from 2-day cultures grown in potato dextrose broth (PDB) at $25 \pm 2{ }^{\circ} \mathrm{C}, 150 \mathrm{rpm}$. These were treated with $p$-anisaldehyde at $0,1 / 2 \mathrm{MIC}$ and MIC for $2 \mathrm{~h}$, respectively. The mycelia morphologies of the samples were directly obtained with a JSM-6610LV scanning electron microscope (JEOL, Japan).

\section{Membrane Permeability Assays}

The membrane permeability of mycelia was analyzed by the propidium iodide (PI) staining method [19]. Twoday-old mycelia from PDB were collected and treated with $p$-anisaldehyde $(0,1 / 2 \mathrm{MIC}$, and MIC) for 30,60 , and $120 \mathrm{~min}$. The treated mycelia were dyed with $10 \mu \mathrm{g} / \mathrm{ml}$ PI for $5 \mathrm{~min}$ at $30^{\circ} \mathrm{C}$, then centrifuged at $4,000 \times g$ for $15 \mathrm{~min}$ after being washed three times with PBS. The mycelia were photographed with an Eclipse TS100 epifluorescence microscope (Nikon Corporation, Japan). The fluorescence values were determinated by a F97 PRO fluorescence spectrophotometer (Lengguang Technology, China) with an excitation wavelength of $535 \mathrm{~nm}$ and the emission wavelength at $615 \mathrm{~nm}$. The results were expressed as the fluorescence value folds.

\section{Determination of Extracellular Alkaline Phosphatase (AKP) Activity}

Two-day-old mycelia from PDB were collected and treated with $p$-anisaldehyde (0, 1/2 MIC, and MIC) for 30, 60 , and $120 \mathrm{~min}$. The extracellular AKP activity was determined by a UV-2450 UV/Vis spectrophotometer (Shimadzu Co., Ltd., China) according to the instructions for the Alkaline Phosphatase (AKP/ALP) Assay Kit (Beijing Solarbio Science \& Technology Co., Ltd., China). All of the tests were performed in triplicate.

\section{Statistical Analysis}

Each parameter was performed in triplicate. All data were analyzed using the SPSS statistical software (Version 16.0) (SPSS Inc., USA). The data were expressed as the mean \pm SD (standard deviation) by measuring three independent replicates and analyzing them by the least significant difference (LSD) test of variance (ANOVA). A value of $p<0.05$ was considered statistically significant. 
Table 1. Effects of $p$-anisaldehyde on mycelial growth of $P$. digitatum.

\begin{tabular}{cccccc}
\hline \multirow{2}{*}{$\begin{array}{c}\text { Concentration } \\
(\mu \mathrm{l} / \mathrm{ml})\end{array}$} & $1 \mathrm{~d}$ & $2 \mathrm{~d}$ & $3 \mathrm{~d}$ & $4 \mathrm{~d}$ & $5 \mathrm{~d}$ \\
\cline { 2 - 6 } & $14.3 \pm 0.0 \mathrm{~d}$ & $13.2 \pm 2.5 \mathrm{e}$ & $10.5 \pm 2.6 \mathrm{~d}$ & $7.3 \pm 2.1 \mathrm{e}$ & $11.5 \pm 2.8 \mathrm{e}$ \\
0.12 & $45.7 \pm 4.9 \mathrm{c}$ & $22.2 \pm 2.5 \mathrm{~d}$ & $18.4 \pm 2.6 \mathrm{~d}$ & $26.2 \pm 2.8 \mathrm{~d}$ & $24.4 \pm 2.0 \mathrm{~d}$ \\
0.25 & $62.9 \pm 4.9 \mathrm{~b}$ & $64.7 \pm 4.4 \mathrm{c}$ & $46.5 \pm 1.5 \mathrm{c}$ & $34.1 \pm 3.2 \mathrm{c}$ & $31.1 \pm 4.3 \mathrm{c}$ \\
0.50 & $100.0 \pm 0.0 \mathrm{a}$ & $75.7 \pm 3.1 \mathrm{~b}$ & $57.9 \pm 3.7 \mathrm{~b}$ & $47.9 \pm 1.3 \mathrm{~b}$ & $40.7 \pm 4.7 \mathrm{~b}$ \\
1.00 & $100.0 \pm 0.0 \mathrm{a}$ & $100.0 \pm 0.0 \mathrm{a}$ & $100.0 \pm 0.0 \mathrm{a}$ & $100.0 \pm 0.0 \mathrm{a}$ & $100.0 \pm 0.0 \mathrm{a}$ \\
2.00 & $100.0 \pm 0.0 \mathrm{a}$ & $100.0 \pm 0.0 \mathrm{a}$ & $100.0 \pm 0.0 \mathrm{a}$ & $100.0 \pm 0.0 \mathrm{a}$ & $100.0 \pm 0.0 \mathrm{a}$ \\
4.00 & &
\end{tabular}

${ }^{*}$ The presented data are the means \pm standard error of pooled data $(n=3)$. Columns with different letters at each time point indicate significant differences according to LSD test at $p<0.05$, the same as below.

Table 2. Effects of $p$-anisaldehyde on mycelial growth of $P$. italicum.

\begin{tabular}{cccccc}
\hline \multirow{2}{*}{$\begin{array}{c}\text { Concentration } \\
(\mu \mathrm{l} / \mathrm{ml})\end{array}$} & \multicolumn{5}{c}{ Inhibitory rate $(\%)$} \\
\cline { 2 - 6 } & $0.0 \pm 0.7 \mathrm{c}$ & $24.1 \pm 6.4 \mathrm{c}$ & $29.8 \pm 6.4 \mathrm{~d}$ & $42.4 \pm 6.7 \mathrm{c}$ & $34.5 \pm 10.0 \mathrm{c}$ \\
\hline 0.12 & $6.3 \pm 1.8 \mathrm{c}$ & $33.3 \pm 11.1 \mathrm{c}$ & $36.2 \pm 8.4 \mathrm{~cd}$ & $36.5 \pm 13.3 \mathrm{c}$ & $28.2 \pm 11.6 \mathrm{c}$ \\
0.25 & $62.5 \pm 0.0 \mathrm{~b}$ & $35.2 \pm 16.0 \mathrm{c}$ & $44.7 \pm 13.3 \mathrm{c}$ & $45.8 \pm 11.0 \mathrm{c}$ & $34.0 \pm 11.0 \mathrm{c}$ \\
0.50 & $100.0 \pm 0.0 \mathrm{a}$ & $63.0 \pm 3.2 \mathrm{~b}$ & $70.2 \pm 3.7 \mathrm{~b}$ & $73.2 \pm 3.2 \mathrm{~b}$ & $69.1 \pm 1.8 \mathrm{~b}$ \\
1.00 & $100.0 \pm 0.0 \mathrm{a}$ & $100.0 \pm 0.0 \mathrm{a}$ & $100.0 \pm 0.0 \mathrm{a}$ & $100.0 \pm 0.0 \mathrm{a}$ & $100.0 \pm 0.0 \mathrm{a}$ \\
2.00 & $100.0 \pm 0.0 \mathrm{a}$ & $100.0 \pm 0.0 \mathrm{a}$ & $100.0 \pm 0.0 \mathrm{a}$ & $100.0 \pm 0.0 \mathrm{a}$ & $100.0 \pm 0.0 \mathrm{a}$ \\
4.00 & & & & &
\end{tabular}

\section{Results}

Effects of $p$-Anisaldehyde on Mycelial Growth

The effects of $p$-anisaldehyde on the mycelial growth of $P$. digitatum and $P$. italicum are shown in Tables 1 and 2. The in vitro assay results on agar plates indicate the mycelial growth of $P$. digitatum and $P$. italicum was markedly $(p$ $<0.05)$ inhibited in a dose-dependent manner by $p$-anisaldehyde. After $5 \mathrm{~d}$ of culture, about $11.5 \pm 2.8 \%$ and 34.5 $\pm 10.0 \%$ of the $P$. digitatum and $P$. italicum mycelia growths were inhibited by $0.12 \mu \mathrm{l} / \mathrm{ml}$ of $p$-anisaldehyde. When the concentration of $p$-anisaldehyde reached $2.00 \mu \mathrm{l} / \mathrm{ml}$, the mycelia showed no visible growth until 4 days of culture. Therefore, the MIC and MFC of $p$-anisaldehyde against $P$. digitatum and $P$. italicum were both estimated to be $2.00 \mu \mathrm{l} / \mathrm{ml}$.

\section{Effects of $p$-Anisaldehyde on Disease Incidence}

The ability of $p$-anisaldehyde to inhibit the green mold of citrus fruit inoculated with $P$. digitatum is shown in Table 3 . The fruit in control group began to rot after $3 \mathrm{~d}$ of storage, and the disease incidence was significantly $(p<$ $0.05)$ delayed in $p$-anisaldehyde treatment in a dose-dependent manner. The control fruit disease incidence was $30 \pm 10 \%$ at 5 days of storage, whereas this percentage of disease incidence in citrus fruits treated with $p$ anisaldehyde (MFC) was reduced to $10 \pm 10 \%$. With prolonged storage time, the disease incidence of green mold increased. After 7 days of storage, the green mold incidence of control fruits was $100 \%$ while that of $p$ anisaldehyde-treated $(1 \times$ MFC and $10 \times$ MFC) fruits was only $90 \pm 8.6 \%$ and $45 \pm 5 \%$, respectively.

Similarly, the blue mold development of citrus fruit inoculated with $P$. italicum was inhibited by $p$-anisaldehyde treatment (Table 4). The control fruit began to rot after $1 \mathrm{~d}$ of storage. After 8 days of storage, the disease incidence of the control fruits was $35 \pm 0 \%$, and it was still $0 \pm 0 \%$ in citrus fruits treated with $p$-anisaldehyde (MFC and $10 \times$ MFC). The blue mold incidence in the control fruits was $100 \%$ after 12 days of storage, and the incidence of $p$ anisaldehyde-treated (MFC and $10 \times$ MFC) fruits was only $25 \pm 10 \%$ and $5 \pm 0 \%$ respectively. The inhibitory effect of $p$-anisaldehyde on blue mold incidence was higher than that on green mold.

Table 3. Green mold incidence in inoculated fruit treated with $p$-anisaldehyde during storage.

\begin{tabular}{cccc}
\hline Day & \multicolumn{3}{c}{ Disease incidence (\%) } \\
\cline { 2 - 4 } & CK & MFC & $10 \times$ MFC \\
2 & $0 \pm 0$ & $0 \pm 0$ & $0 \pm 0$ \\
3 & $0 \pm 0$ & $0 \pm 0$ & $0 \pm 0$ \\
4 & $5 \pm 5$ & $0 \pm 0$ & $0 \pm 0$ \\
5 & $30 \pm 10$ & $10 \pm 10$ & $20 \pm 5$ \\
6 & $60 \pm 10$ & $60 \pm 5$ & $35 \pm 8.6$ \\
7 & $90 \pm 5$ & $85 \pm 8.6$ & $45 \pm 5$ \\
\hline
\end{tabular}

*The inoculated fruits of CK were sprayed with wax only, and inoculated fruits of MFC or $10 \times$ MFC were sprayed with $p$ anisaldehyde wax at 2.0 or $20.0 \mu \mathrm{l} / \mathrm{ml}$, the same as below. 
Table 4. Blue mold incidence in inoculated fruit treated with $p$-anisaldehyde during storage.

\begin{tabular}{cccc}
\hline \multirow{2}{*}{ Day } & \multicolumn{3}{c}{ Disease incidence (\%) } \\
\cline { 2 - 4 } & CK & MFC & $0 \pm 0$ \\
2 & $5 \pm 0$ & $0 \pm 0$ & $0 \pm 0$ \\
3 & $10 \pm 5$ & $0 \pm 0$ & $0 \pm 0$ \\
4 & $10 \pm 5$ & $0 \pm 0$ & $0 \pm 0$ \\
5 & $10 \pm 5$ & $0 \pm 0$ & $0 \pm 0$ \\
6 & $10 \pm 5$ & $0 \pm 0$ & $0 \pm 0$ \\
7 & $10 \pm 5$ & $0 \pm 0$ & $0 \pm 0$ \\
8 & $20 \pm 5$ & $0 \pm 0$ & $0 \pm 0$ \\
9 & $35 \pm 0$ & $5 \pm 0$ & $5 \pm 0$ \\
10 & $45 \pm 5$ & $10 \pm 0$ & $5 \pm 0$ \\
11 & $60 \pm 0$ & $10 \pm 0$ & $5 \pm 0$ \\
\hline
\end{tabular}

\section{Scanning Electron Microscopy}

The effects of $p$-anisaldehyde on the morphology of $P$. digitatum and $P$. italicum mycelia were examined by SEM (Fig. 1). As shown in Figs. 1A-1 and 1B-1, the control samples had a tubular, regular, full shape, with homogeneous and smooth surface morphology. In contrast, the 1/2 MIC of $p$-anisaldehyde-treated mycelia showed irregular, wizened, rough and collapsed surfaces (Figs. $1 \mathrm{~A}-2$ and $1 \mathrm{~B}-2$ ). This phenomenon was more apparent as the concentration of $p$-anisaldehyde increased. As revealed by Figs. 1A-3 and 1B-3, the MIC $p$-anisaldehyde-treated mycelia became flat, widened, and wrinkled with irregular structures and shapes.

\section{Membrane Permeability Assay}

The effects of $p$-anisaldehyde on the membrane permeability of $P$. digitatum and $P$. italicum are shown in Figs. 2 and 3 . After $30 \mathrm{~min}$ of exposure, the fluorescence intensities of all treatments in $P$. digitatum samples were decreased (Fig. 2A). However, the fluorescence values in $P$. digitatum treated with $1 / 2$ MIC and MIC of $p$ anisaldehyde were $1.32 \pm 0.09$ and $1.20 \pm 0.05$ times that of the control at $60 \mathrm{~min}$ of incubation. With time prolonged to $120 \mathrm{~min}$, the fluorescence value of $P$. digitatum in MIC $p$-anisaldehyde treatment was nearly $1.41 \pm$ 0.09 times higher than that of the control. With regard to $P$. italicum, the fluorescence values of the $p$-anisaldehydetreated mycelia were significantly higher $(p<0.05)$ than control groups during the whole period (Fig. 2B). The fluorescence values of the MIC $p$-anisaldehyde-treated $P$. italicum were approximately $1.53 \pm 0.16,1.49 \pm 0.06,1.46$ \pm 0.12 times higher than that of control at 30,60 , and 120 min of exposure.

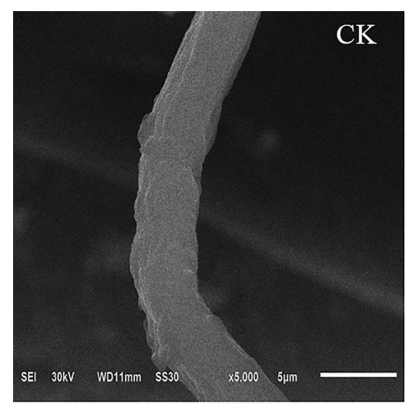

A-1

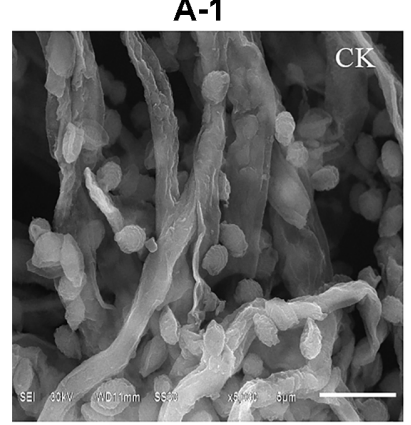

B-1

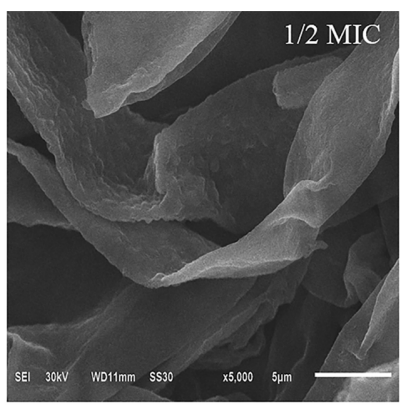

A-2

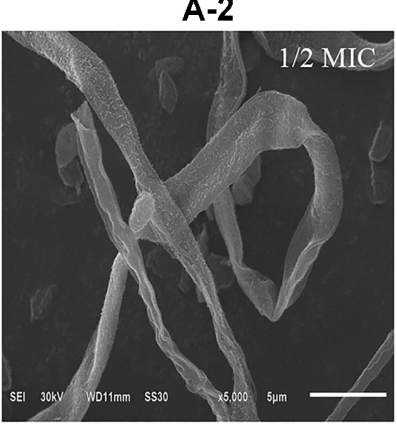

B-2

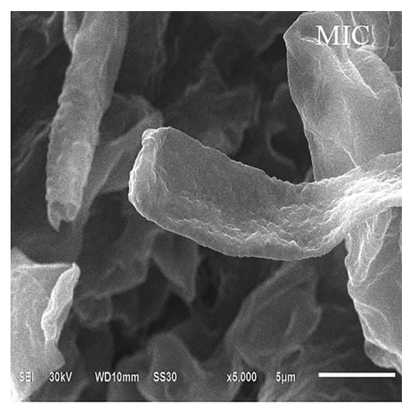

A-3

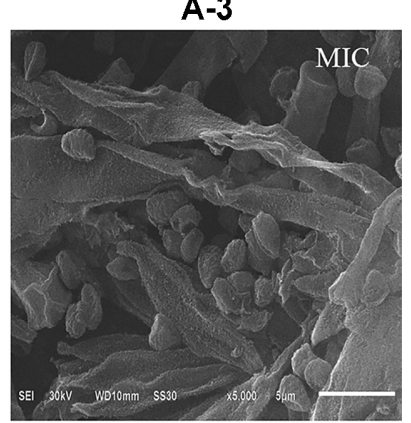

B-3

Fig. 1. SEM images of $P$. digitatum (A-1, A-2, A-3) and P. italicum (B-1, B-2, B-3) mycelial morphology after p-anisaldehyde treatment. Untreated hyphae (A-1, B-1); treatment with $1 / 2$ MIC of $p$-anisaldehyde (A-2, B-2); treatment with MIC of $p$-anisaldehyde (A-3, B-3) $(\mathrm{bar}=5 \mu \mathrm{m})$. 

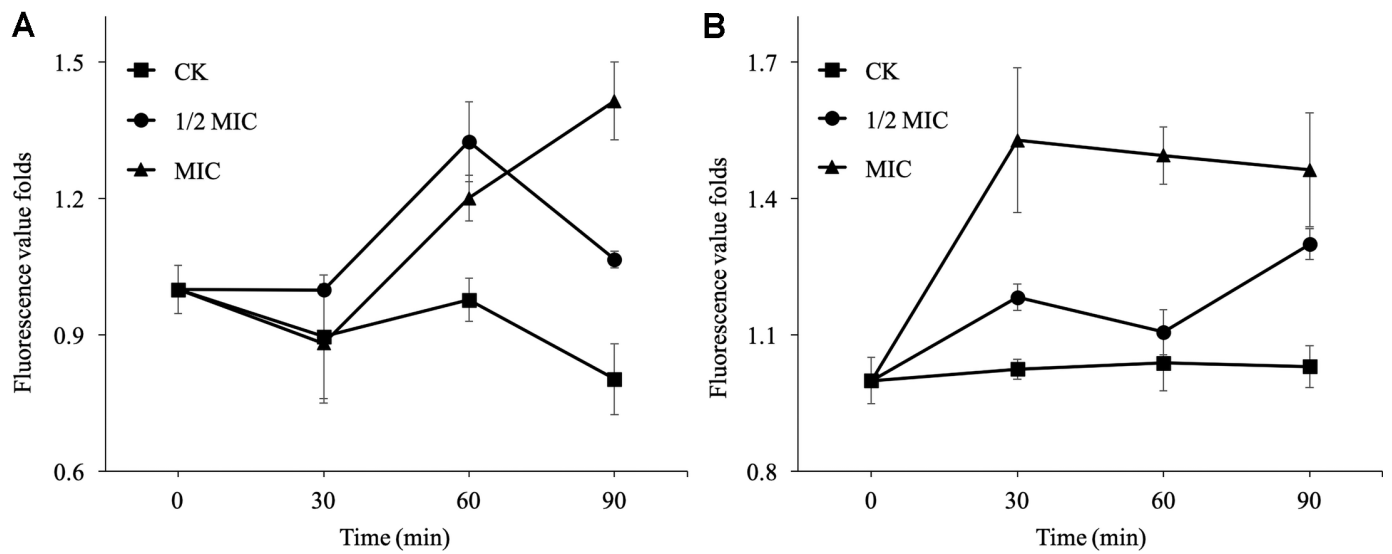

Fig. 2. Effects of $p$-anisaldehyde treatment on the PI fluorescence fold changes of P. digitatum (A) and P. italicum (B) mycelia. Data presented are the means of the pooled data. Error bars indicate the SDs of the means $(n=3)$, the same as below.

The fluorescence microscopy images of the mycelium treated with $p$-anisaldehyde are shown in Fig. 3 . The results were consistent with fluorescence values described above. There was no visible red fluorescence observed in CK after 120 min of incubation. In the P. digitatum mycelium treated with $1 / 2 \mathrm{MIC}$ and MIC, red fluorescence was exclusively observed after $60 \mathrm{~min}$ of exposure (Fig. 3A). And in the P. italicum hyphae treated with MIC, the red fluorescence was observed after 30 min of exposure (Fig. 3B).

A

$30 \min$

$60 \min$

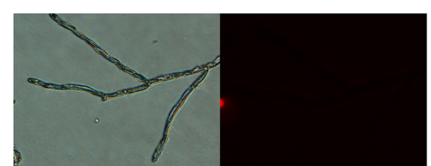

$120 \mathrm{~min}$
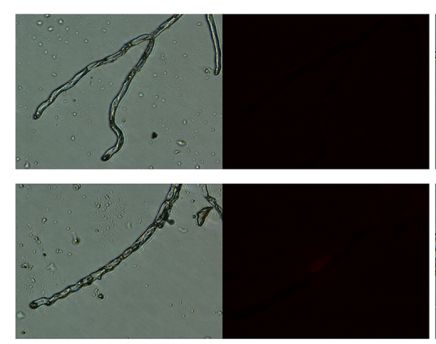

Brightfield

PI
B
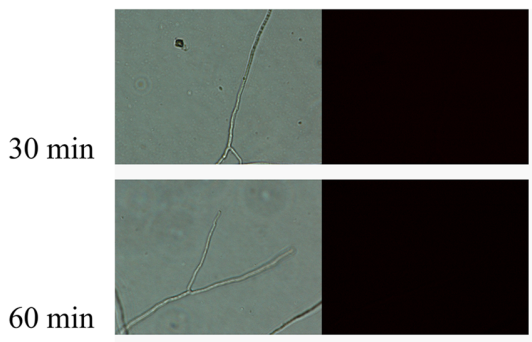

$60 \mathrm{~min}$

$120 \mathrm{~min}$

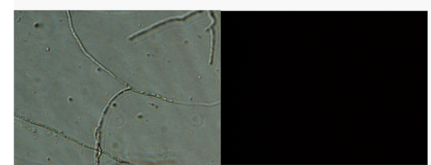

Brightfield
PI
1/2 MIC
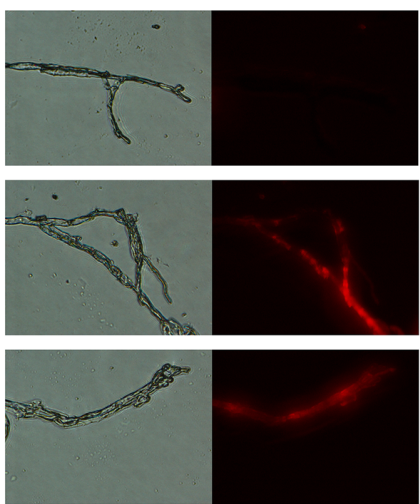

Brightfield
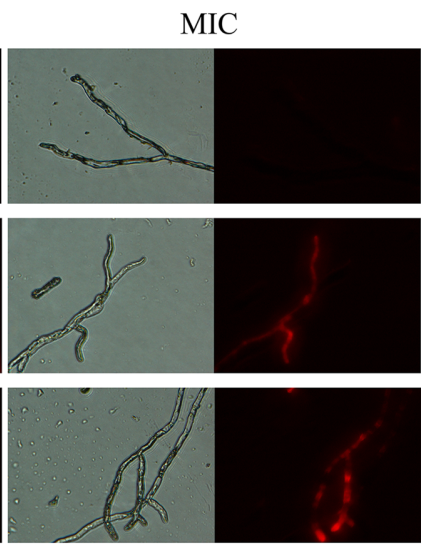

Brightfield

PI

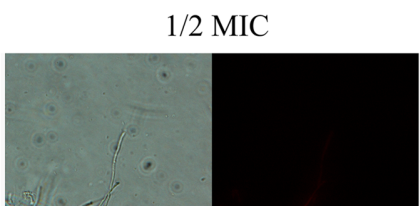

MIC
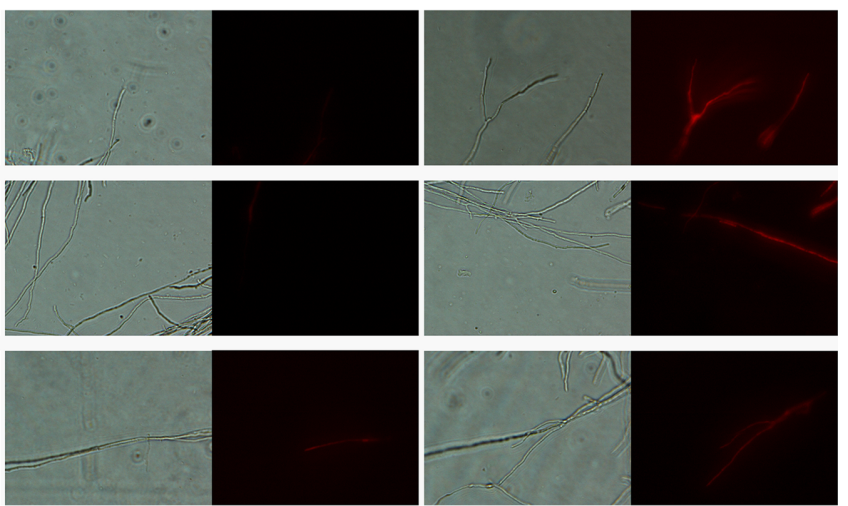

Brightfield

PI

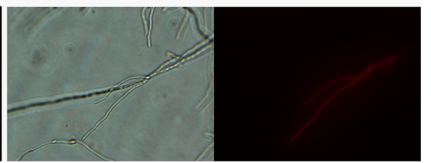

Brightfield

PI

Fig. 3. Effects of $p$-anisaldehyde treatment on the plasma membrane integrity of $P$. digitatum (A) and $P$. italicum (B) mycelia. 

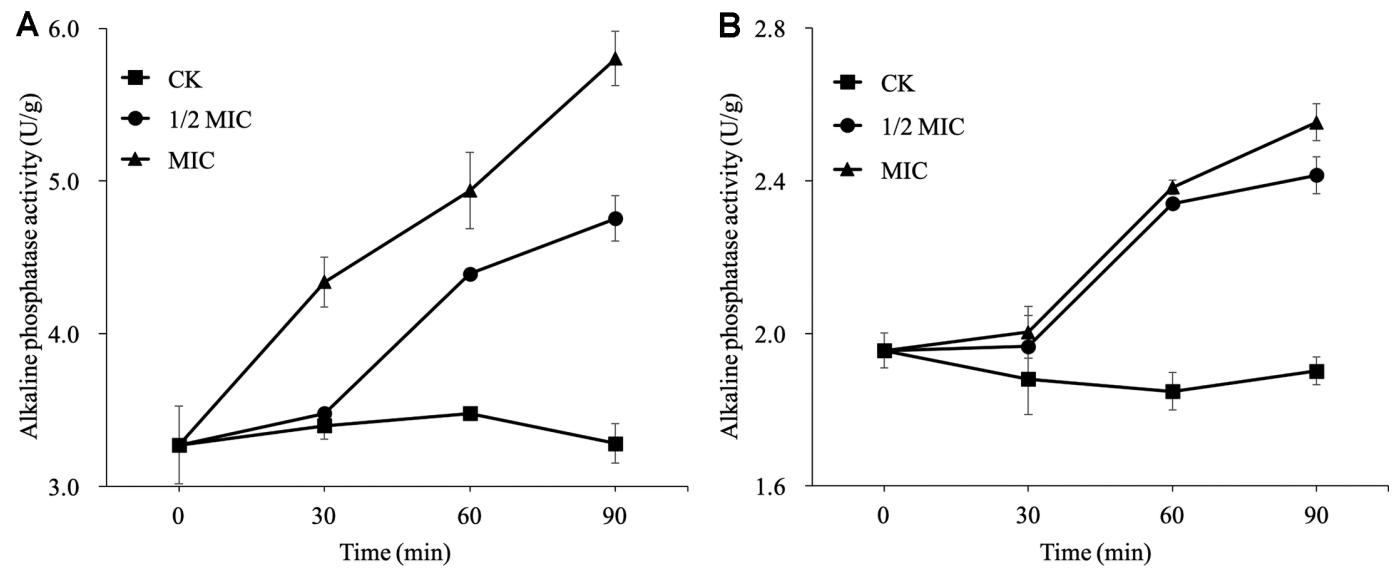

Fig. 4. Effects of $p$-anisaldehyde treatment on the AKP activity of $P$. digitatum (A) and P. italicum (B).

\section{Determination of Extracellular Alkaline Phosphatase (AKP) Activity}

The effects of $p$-anisaldehyde (0,1/2 MIC and MIC) on the extracellular AKP activity of $P$. digitatum and $P$. italicum were shown in Fig. 4. The extracellular AKP activity in 1/2 MIC and MIC $p$-anisaldehyde-treated $P$. digitatum and $P$. italicum mycelia were all higher than that of control. The extracellular AKP activity in MIC $p$ anisaldehyde-treated $P$. digitatum was higher than that of $1 / 2$ MIC treatment during the whole period. After 120 min of treatment, the extracellular AKP activity of $P$. digitatum mycelia treated with MIC of $p$-anisaldehyde was $5.80 \pm 0.18 \mathrm{U} / \mathrm{g}$, which was significantly higher than the control $(3.27 \pm 0.13 \mathrm{U} / \mathrm{g})$ (Fig. $4 \mathrm{~A})$. The extracellular AKP activity levels of both 1/2 MIC- and MIC-treated P. italicum showed the same variation trend during the whole incubation period (Fig. 4B). The extracellular AKP activity of $P$. italicum treated with $1 / 2$ MIC and MIC $p$ anisaldehyde increased and reached $2.41 \pm 0.05$ and $2.55 \pm 0.05 \mathrm{U} / \mathrm{g}$ after $120 \mathrm{~min}$ of treatment, respectively, which was significantly higher than the control $(1.90 \pm 0.04 \mathrm{U} / \mathrm{g})$.

\section{Discussion}

Essential oils as a replacement for synthetic fungicides are widely used for controlling postharvest diseases [9, 20-22]. $p$-Anisaldehyde, primarily isolated from anise, also exhibits strong antifungal activity against a number of yeast and mold strains $[14,23,24]$. Shreaz et al. indicated the MIC values of $p$-anisaldehyde for Candida (fluconazole-resistant strains and fluconazole-sensitive laboratory strains) ranged from 250 to $600 \mu \mathrm{g} / \mathrm{ml}$ [12]. The MICs of $p$-anisaldehyde for S. cerevisiae and S. aureus were found to be $256 \mu \mathrm{g} / \mathrm{ml}$ [16] and $2 \sim 4 \mathrm{mg} / \mathrm{ml}$ [15] respectively. In this research, $p$-anisaldehyde was observed to elicit strong antifungal activity against $P$. digitatum and $P$. italicum, with the MIC and MFC values of both being $2.00 \mu \mathrm{l} / \mathrm{ml}$, which were much similar with those of the microorganism mentioned above. Furthermore, in vivo assays demonstrated that $p$-anisaldehyde visibly inhibited both the green mold and blue mold development of citrus fruits inoculated with P. digitatum and P. italicum.

Cell membranes play the vital role of protecting the intracellular substances and maintaining cell viability, and lack of membrane integrity can result in cell death $[25,26]$. Many studies have indicated that essential oils such as those extracted from palmarosa, tea tree, thyme, and star anise could inhibit the growth of microorganisms by breaking the membrane integrity $[11,27,28]$. After $p$-anisaldehyde-treatment for $120 \mathrm{~min}$, the mycelia morphology of $P$. digitatum and $P$. italicum was greatly altered. And the SEM results (Fig. 1 ) showed that the $p$ anisaldehyde-treated mycelia were collapsed and even fractured. The PI staining showed the mycelia of $P$. digitatum and $P$. italicum were stained with PI after $p$-anisaldehyde treatment, suggesting that the membrane integrity of $P$. digitatum and $P$. italicum mycelia was disrupted (Fig. 3).

AKP activity is generally used as the parameter indicating the cell wall integrity $[29,30]$. In previous studies, essential oils from tea tree, Marisela minuta, and wild blueberry were reported to induce the increase in the AKP activity and thus damaged the cell wall integrity of Botrytis cinerea, Pseudomonas aeruginosa and various foodborne pathogens $[21,31,32]$. In our previous research, calcofluor white staining and AKP activity were used for the cell wall integrity together. And the experimental results show that these two methods are in agreement with each other [33]. So, AKP activity was used to indicate the cell wall integrity in this research. The results showed that the extracellular AKP activity in 1/2 MIC and MIC $p$-anisaldehyde-treated $P$. digitatum and P. italicum mycelia were all significantly $(p<0.05)$ higher than that of control (Fig. 4$)$. Those results suggested that the impairment in the cell wall integrity might be highly involved in the inhibition of $p$-anisaldehyde on $P$. digitatum and $P$. italicum.

In conclusion, our present study indicated that $p$-anisaldehyde exhibited strong antifungal activity against mycelia growths of $P$. digitatum and $P$. italicum. The inhibitory mechanism involved might have to do with the destruction of the cell wall integrity and membrane permeability. 


\section{Acknowledgments}

This project was supported by the National Natural Science Foundation of China (Grant number 31801603), the Research Foundation of the Education Bureau of Hunan Province (No. 19A476), the Natural Science Fund of Hunan Province of China (2019JJ50580), and the Xiangtan University Scientific Research Foundation for PhD (Grant numbers 18QDZ04).

\section{Conflict of Interest}

The authors have no financial conflicts of interest to declare.

\section{References}

1. Papoutsis K, Mathioudakis MM, Hasperue JH, Ziogas V. 2019. Non-chemical treatments for preventing the postharvest fungal rotting of citrus caused by Penicillium digitatum (green mold) and Penicillium italicum (blue mold). Trends Food Sci. Technol. 86: 479-491.

2. Droby S, Eick A, Macarisin D, Cohen L, Rafael G, Stange R, et al. 2008. Role of citrus volatiles in host recognition, germination and growth of Penicillium digitatum and Penicillium italicum. Postharvest Biol. Technol. 49: 386-396.

3. Zhu C, Lei M, Andargie M, Zeng J, Li J. 2019. Antifungal activity and mechanism of action of tannic acid against Penicillium digitatum. Physiol. Mol. Plant Pathol. 107: 46-50.

4. Li L, Tang X, Ouyang Q, Tao N. 2019. Combination of sodium dehydroacetate and sodium silicate reduces sour rot of citrus fruit. Postharvest Biol. Technol. 151: 19-25.

5. Macarisin D, Cohen L, Eick A, Rafael G, Belausov E, Wisniewski M, et al. 2007. Penicillium digitatum suppresses production of hydrogen peroxide in host tissue during infection of citrus fruit. Phytopathology 97: 1491-1500.

6. Berk Z. 2016. Chapter 6 - Postharvest changes. Citrus Fruit Processing.

7. Hao W, Hui L, Hu M, Liu Y, Rizwan-Ul-Haq M. 2011. Integrated control of citrus green and blue mold and sour rot by Bacillus amyloliquefaciens in combination with tea saponin. Postharvest Biol. Technol. 59: 316-323.

8. Sancheztorres, Paloma, Tuset, Juan J. 2011. Molecular insights into fungicide resistance in sensitive and resistant Penicillium digitatum strains infecting citrus. Postharvest Biol.Technol. 59: 159-165.

9. Simas DLR, de Amorim SHBM, Goulart FRV, Alviano CS, Alviano DS, da Silva AJR. 2017. Citrus species essential oils and their components can inhibit or stimulate fungal growth in fruit. Ind. Crops Prod. 98: 108-115.

10. Chen CY, Cai N, Chen JY, Wan CP. 2019. Clove essential oil as an alternative approach to control postharvest blue mold caused by Penicillium italicum in Citrus Fruit. Biomolecules 9: 197.

11. Zhang J, Wu H, Jiang D, Yang Y, Tang W, Xu K. 2019. The antifungal activity of essential oil from Melaleuca leucadendra (L.) L. grown in China and its synergistic effects with conventional antibiotics against Candida. Nat. Prod. Res. 33: 2545-2548.

12. Shreaz S, Bhatia R, Khan N, Muralidhar S, Basir SF, Manzoor N, et al. 2011. Exposure of Candida to $p$-anisaldehyde inhibits its growth and ergosterol biosynthesis. J. Gen. Appl. Microbiol. 57: 129-136.

13. Showler AT, Harlien JL. 2019. Lethal and repellent effects of the botanical p-anisaldehyde on Musca domestica (Diptera: Muscidae). J. Econ. Entomol. 112: 485-493.

14. Chen X, Zhang X, Meng R, Zhao Z, Liu Z, Zhao X, et al. 2016. Efficacy of a combination of nisin and p-anisaldehyde against Listeria monocytogenes. Food Control 66: 100-106.

15. Shi C, Zhao X, Meng R, Liu Z, Zhang G, Guo N. 2017. Synergistic antimicrobial effects of nisin and p-anisaldehyde on Staphylococcus aureus in pasteurized milk. Lwt-Food Sci. Technol. 84: 222-230.

16. Yu L, Guo N, Yang Y, Wu X, Meng R, Fan J, et al. 2010. Microarray analysis of $p$-anisaldehyde-induced transcriptome of Saccharomyces cerevisiae. J. Ind. Microbiol. Biotechnol. 37: 313-322.

17. Tao N, Fan F, Jia L, Zhang M. 2014. Octanal incorporated in postharvest wax of Satsuma mandarin fruit as a botanical fungicide against Penicillium digitatum. Food Control 45: 56-61.

18. Dou S, Ouyang Q, You K, Qian J, Tao N. 2018. An inclusion complex of thymol into beta-cyclodextrin and its antifungal activity against Geotrichum citri-aurantii. Postharvest Biol. Technol. 138: 31-36.

19. Liu J, Zong YY, Qin GZ, Li BQ, Tian SP. 2010. Plasma membrane damage contributes to antifungal activity of silicon against Penicillium digitatum. Curr. Microbiol. 61: 274-279.

20. Talibi I, Askarne L, Boubaker H, Boudyach EH, Msanda F, Saadi B, et al. 2012. Antifungal activity of Moroccan medicinal plants against citrus sour rot agent Geotrichum candidum. Lett. Appl. Microbiol. 55: 155-161.

21. Shao X, Cheng S, Wang H, Yu D, Mungai C. 2013. The possible mechanism of antifungal action of tea tree oil on Botrytis cinerea. J. Appl. Microbiol. 114: 1642-1649.

22. Regnier T, Combrinck S, Veldman W, Du Plooy W. 2014. Application of essential oils as multi-target fungicides for the control of Geotrichum citri-aurantii and other postharvest pathogens of citrus. Ind. Crops Prod. 61: 151-159.

23. Neri F, Mari M, Brigati S, Bertolini P. 2009. Control of Neofabraea alba by plant volatile compounds and hot water. Postharvest Biol. Technol. 51: 425-430.

24. Sharopov F, Valiev A, Satyal P, Gulmurodov I, Yusufi S, Setzer WN, et al. 2017. Cytotoxicity of the essential oil of fennel (Foeniculum vulgare) from Tajikistan. Foods 6: 73 .

25. Shao X, Cao B, Xu F, Xie S, Yu D, Wang H. 2015. Effect of postharvest application of chitosan combined with clove oil against citrus green mold. Postharvest Biol. Technol. 99: 37-43.

26. Liu K, Zhou X, Fu M. 2017. Inhibiting effects of epsilon-poly-lysine (ع-PL) on Pencillium digitatum and its involved mechanism. Postharvest Biol. Technol. 123: 94-101.

27. Kang J, Liu L, Wu X, Sun Y, Liu Z. 2018. Effect of thyme essential oil against Bacillus cereus planktonic growth and biofilm formation. Appl.Microbiol. Biotechnol. 102: 10209-10218.

28. da Rocha Neto AC, Navarro BB, Canton L, Maraschin M, Di Piero RM. 2019. Antifungal activity of palmarosa (Cymbopogon martinii), tea tree (Melaleuca alternifolia) and star anise (Illicium verum) essential oils against Penicillium expansum and their mechanisms of action. Lwt-Food Sci. Technol. 105:385-392.

29. Yang S, Liu L, Li D, Xia H, Su X, Peng L, et al. 2016. Use of active extracts of poplar buds against Penicillium italicum and possible modes of action. Food Chem. 196: 610-618.

30. Lengeler KB, Wasserstrom L, Walther A, Wendland J. 2013. Analysis of the cell wall integrity pathway of Ashbya gossypii. Microbiol. Res. 168: 607-614.

31. Arokiyaraj S, Bharanidharan R, Agastian P, Shin H. 2018. Chemical composition, antioxidant activity and antibacterial mechanism of action from Marsilea minuta leaf hexane: methanol extract. Chem. Cent. J. 12: 105.

32. Sun X, Zhou T, Wei C, Lan W, Zhao Y, Pan Y, et al. 2018. Antibacterial effect and mechanism of anthocyanin rich Chinese wild blueberry extract on various foodborne pathogens. Food Control 94: 155-161.

33. OuYang QL, Duan XF, Li L, Tao NG. 2019. Cinnamaldehyde exerts its antifungal activity by disrupting the cell wall integrity of Geotrichum citri-aurantii. Front.Microbiol. 10: 9. 